\title{
Diagnóstico de salud poblacional: Lucena del Cid
}

\author{
MIRIAM IZQUIERDO MARTÍNEZ \\ al339178@uji.es \\ AITOR LUJÁN RONDA \\ al343695@uji.es \\ Águeda Cervera Gasch \\ cerveraa@uji.es \\ PABlo Salas MEdina \\ psalas@uji.es
}

\section{Resumen}

Introducción: Lucena del Cid es una población de montaña que basa su economía en la industria y servicios, y con una población de 1417 habitantes con una distribución de $50,2 \%$ de hombres y 49,82 \% de mujeres. La tasa de natalidad de la población es del $2,12 \%$ y la tasa de mortalidad del $19,05 \%$. Cuenta con un centro de salud con referencia de especialidades y hospital a $45 \mathrm{~km}$. Metodología: Se desarrolla un estudio observacional-descriptivo-transversal, realizado por alumnos de segundo de Enfermería de la Universitat Jaume I, entre los meses de noviembre de 2016 y enero de 2017. Para llevar a cabo dicho estudio, se han utilizado tanto fuentes de documentación primarias como fuentes secundarias. A su vez se realizó una búsqueda de datos estadísticos en el Instituto Nacional de Estadística y en Instituto Valenciano de Estadístico. Resultados: La primera causa de mortalidad son las enfermedades del sistema circulatorio $(27 \%)$ seguida de neoplasias $(23 \%)$ y enfermedades respiratorias (8 $\%)$. El nivel de dependencia juvenil es del $17,1 \%$ y de un $42,9 \%$ en ancianos. La población activa es de 418 personas distribuidas en un 38,03 \% de mujeres y un 61,96 $\%$ de hombres. Conclusiones: Lucena tiene una población longeva (53 \%) y con elevadas tasas de dependencia (60\%). Es necesaria una restructuración de los servicios sanitarios, facilitando la cobertura de las necesidades de una población envejecida.

Palabras clave: diagnóstico, salud, poblacional, Lucena del Cid, descriptivo

\begin{abstract}
Introduction: Lucena del $\mathrm{Cid}$ is a mountain village that bases its economy on industry and services. It has a population of 1417 inhabitants with a distribution of $50.2 \%$ males and $49.82 \%$ females. The birth rate of its population is $2.12 \%$ and it has a mortality rate of $19.05 \%$. Lucena del Cid has a health centre with reference of specialties and a hospital $45 \mathrm{~km}$ away. Methodology: It is an observational-descriptive-transversal study carried out by students of the Nursing Department of the Universitat Jaume I, between November 2016 and January 2017. In order to carry out this research study,
\end{abstract}


both primary and secondary sources of documentation were used. In turn, we carried out a search of statistical data in the National Institute of Statistics and in the Valencian Institute of Statistics. Results: The first cause of mortality are diseases of the circulatory system (27\%) followed by neoplasias (23\%), and respiratory diseases (8\%). The level of juvenile dependency is $17.1 \%$ and $42.9 \%$ in the elderly. The active population (418 people) is distributed in $38.03 \%$ of women and $61.96 \%$ of men. Conclusion: Lucena has an aged population (53\%) and with high dependency rates (60\%). There is a need for a restructuring of health services, facilitating the coverage of the needs of an aging population.

Keywords: diagnosis, health, population, Lucena del Cid, descriptive.

\section{Introducción}

Un diagnóstico de salud es un estudio que tiene como objetivo conocer cuál es el estado de salud de una población mediante el análisis exhaustivo de sus principales problemas de salud y las necesidades la misma, así como conocer los recursos con los que se cuenta dicha población para solucionar las variables problemáticas. Por tanto, un diagnóstico de salud es el inicio del proceso de planificación de una intervención sanitaria y resulta una herramienta fundamental para la investigación en salud pública (Arenas-Monreal, Cortez-Lugo, ParadaToro, Pacheco-Magaña \& Magaña-Valladares, 2015).

El presente estudio se realizará sobre la población de Lucena del Cid, que se encuentra en el interior de la provincia de Castellón, en la Comunidad Valenciana. Concretamente está situado en la comarca de l'Alcalatén, limitando con la provincia de Teruel (Lucenadelcid.es, 2016). Este diagnóstico de salud tiene como propósito presentar y analizar la situación de la población de Lucena del Cid. Lo que se quiere conseguir con este estudio poblacional de salud es detectar los principales problemas sanitarios en la comunidad, así como proponer soluciones de mejora para cada uno de ellos.

Se eligió este municipio por su geografía, pasado histórico, economía y, sobre todo, por la situación de la población en materia de salud, ya que cuenta con una infraestructura mínima para el desarrollo de las actividades sanitarias.

El objetivo general de este estudio era conocer y caracterizar el estado real de salud del municipio de Lucena del Cid mediante el análisis del estudio estadístico de la población, con el fin de enunciar propuestas de actuación acordes con la realidad de la población.

En cuanto a los objetivos específicos fueron los siguientes:

- Realizar un estudio de los indicadores de salud más relevantes de Lucena del Cid con el fin de obtener datos acerca de la situación actual en la población de estudio.

- Estudiar la situación demográfica, económica y social del municipio, y comprobar si existe alguna relación entre los indicadores de salud que se han obtenido con el estado de salud actual de la población de Lucena del Cid.

- Conocer las enfermedades más prevalentes de dicha población y sus principales causas con el fin de enfocar las pertinentes medidas sanitarias hacia la prevención de dichas enfermedades con el fin de disminuir su incidencia.

- Conocer los recursos sociales y económicos con los que cuenta Lucena del Cid. 
- Estudiar las necesidades y los déficits que tienen los habitantes del municipio en todas las áreas de estudio que se han mencionado anteriormente.

- Plantear programas de salud adaptados a las enfermedades más relevantes de dicha población, en el caso que sea necesario.

\section{Método}

Se desarrolla un estudio observacional-descriptivo-transversal, realizado por alumnos de segundo de Enfermería de la Universitat Jaume I en el espacio temporal comprendido desde noviembre de 2016 a enero de 2017. El lugar que se ha elegido para realizar el estudio es Lucena del Cid, un municipio de la provincia de Castellón. El estudio permitirá valorar el nivel de salud del municipio, así como examinar el tipo de enfermedades en función de la población, en un determinado espacio de tiempo.

Para llevar a cabo dicho estudio, se han utilizado tanto fuentes de documentación primarias como fuentes secundarias. Se realizó una búsqueda de datos estadísticos en el Instituto Nacional de Estadística (INE) y en Instituto Valenciano de Estadístico (IVE) (Conselleria de Sanidad, 2016). Se utilizaron los datos del 2014 puesto que, aunque sí que estaban disponibles los datos del empadronamiento del 2015, los demás datos estadísticos no se encontraban publicados. Además, se contactó varias veces con el punto de atención continuada de Lucena del Cid y se acudió al municipio el día 28 de diciembre de 2016 para obtener más información. Se visitó todo el municipio y se acudió tanto al Ayuntamiento de Lucena del Cid como al punto de atención continuada.

- Punto de atención continuada de Lucena del Cid. Plaza España, 22, 12120 Lucena del Cid, Castellón. Teléfono: 96473820

- Ayuntamiento de Lucena del Cid. Plaza España, 22, 12120 Lucena del Cid, Castellón. Teléfono: 964380001.

Los datos estadísticos, así como las variables objetivo del estudio, han sido obtenidos mediante los institutos estadísticos tanto a nivel nacional como a nivel comunitario, como se ha mencionado anteriormente. Sin embargo, al tratarse de un municipio con una población pequeña, algunos datos no se han podido obtener directamente, por lo que se debió realizar una extrapolación con los datos de Castellón, provincia a la que pertenece Lucena del Cid.

El rango de población del estudio comprende toda la población empadronada en 2014 en Lucena del Cid $(n=1417)$, con independencia de la edad, sexo, procedencia y nivel socioeconómico. Por ende, se excluye a todos aquellos que no se encuentren empadronados en el municipio de Lucena del cid en dicho año.

Las variables objeto de estudio, extraídas del IVE, son las siguientes: tasa de natalidad, tasa de mortalidad, dependencia tanto juvenil como anciana, envejecimiento, longevidad, tasa de maternidad, tendencia de la población, renovación de la población activa, esperanza de vida en el nacimiento, población activa económicamente, padrón municipal y, finalmente, renta per cápita.

Las variables objeto de estudio extrapoladas son: enfermedades más relevantes que causan muerte, a partir de los datos obtenidos de la provincia de Castellón a través del IVE y el PIB, sumatorio de inversión, consumo, gasto y exportaciones netas, obtenidas a partir de la renta per cápita. 


\section{Resultados}

Lucena del Cid cuenta con un total de 1417 personas empadronadas según los últimos datos disponibles en el Instituto Valenciano de Estadística (2014). De estas 1417 personas censadas 711 son de hombres $(50,18 \%)$ y 706 son mujeres $(49,82 \%)$ [5]. El municipio presenta una tasa de natalidad del $2,12 \%$ y una tasa de mortalidad del $19,05 \%$. Como se puede apreciar con los datos anteriores, es mucho mayor la tasa de mortalidad que la de natalidad.

Para poder conocer las enfermedades más relevantes que causan esta elevada cifra de mortalidad, se ha tenido que extrapolar los datos existentes de la provincia de Castellón de 2014, ya que no se dispone de estos datos para Lucena del Cid. Los datos que se obtuvieron fueron los siguientes como se presenta en la Tabla 1.

En cuanto a los indicadores demográficos, cabe destacar que la esperanza de vida al nacimiento en Lucena del Cid en hombres es de 80,32 años y en mujeres 85,12 años, siendo el valor de los hombres mayor que la media española $(80,1$ años) y el de las mujeres menos a la media española (85,6 años) en el año 2014 (INE, 2017).

Para conocer con exactitud las características demográficas de la población, se estudian los indicadores demográficos y muestran los siguientes valores: envejecimiento $(249,7 \%)$, longevidad (53\%). dependencia (60\%), de la cual un $17,1 \%$ es juvenil y un $42,9 \%$ anciana, maternidad $(17,8 \%)$, tendencia $(86,4 \%)$, renovación de población activa $(79,5 \%)$.

Tabla 1

Tasa de mortalidad de diferentes enfermedades

\begin{tabular}{|c|c|c|c|c|}
\hline Enfermedades & Hombres & Mujeres & Total & $\begin{array}{c}\text { Extrapolación a Lucena } \\
\text { del Cid en } \%\end{array}$ \\
\hline Sistema circulatorio & 719 & 887 & 1596 & 27,166 \\
\hline Neoplasias & 853 & 524 & 1377 & 23,438 \\
\hline Sistema respiratorio & 272 & 196 & 468 & 7,946 \\
\hline $\begin{array}{l}\text { Sistema nervioso y órganos de los } \\
\text { sentidos }\end{array}$ & 137 & 220 & 357 & 6,077 \\
\hline $\begin{array}{l}\text { De la cavidad bucal, de las glándulas } \\
\text { salivales y de los maxilares }\end{array}$ & 137 & 112 & 249 & 4,238 \\
\hline Mentales y de comportamiento & 83 & 152 & 235 & 3,9999 \\
\hline Endocrinas, nutricionales y metabólicas & 75 & 105 & 180 & 3,0808 \\
\hline $\begin{array}{l}\text { Causas externas de mortalidad y } \\
\text { morbilidad }\end{array}$ & 104 & 67 & 171 & 2,9106 \\
\hline Del sistema genitourinario & 70 & 68 & 138 & 2,3489 \\
\hline Infecciosas y parasitarias & 36 & 27 & 63 & 1,0723 \\
\hline
\end{tabular}

En lo referente a las migraciones, se observa que ha habido un total de 52 emigraciones, siendo 37 a la Comunidad Valenciana $(71,15 \%), 6$ emigraciones a otras comunidades autónomas de España $(11,53 \%$ y 9 al extranjero $(17,30 \%)$. En cuanto a las inmigraciones, se han registrado un total de 26 , donde 20 de ellas han sido procedentes de la Comunidad Valencia- 
na $(76,92 \%), 5$ de otras comunidades autónomas de España $(19,23 \%)$ y 1 persona del extranjero (3,84\%). Dichos datos son de 2013.

Respecto a los indicadores socioeconómicos y de empleo de la población de Lucena del Cid, se obtiene que hay un total de 130 parados, de estos: 0,8\% pertenecen al sector de la agricultura, 13,8 \% perteneciente de la construcción, 45,4 \% provenientes de las industrias, $36,2 \%$ corresponden al sector servicios, 3,8 \% sin ocupación anterior.

Por otro lado, la población activa económicamente (PAE) es de 876 personas. Hay un total de 418 personas trabajadoras afiliadas a la seguridad social, de los cuales 259 son hombres $(61,96 \%)$ y 159 mujeres $(38,03 \%)$. Estos datos, se han obtenido calculando la media de los cuatro trimestres de 2014, cuyas cifras se encuentran disponibles en el IVE. La renta per cápita de Lucena del Cid asciende a un total de 14.002 euros. Por otra parte, el PIB es de 19.840.834 euros; éste se obtiene al multiplicar la renta per cápita por el número de habitantes en ese año, puesto que la relación de éste y el número de habitantes es la renta per cápita.

Como se ha comentado en la introducción, Lucena del Cid cuenta con un punto de atención continuada el cual depende del departamento de Castellón. El hospital de referencia es el Hospital General Universitario de Castellón el cual se encuentra a 35,1 km del municipio. Además, este departamento cuenta con otros hospitales como el Consorcio Hospitalario Provincial de Castellón y el Hospital la Magdalena, localizados en Castellón de la Plana. Se cuenta también con el centro de especialidades Jaume I, así como el centro de diálisis Nefroplana, que se localizan también en la capital de la provincia. Cabe destacar que un día a la semana pasan por Lucena un fisioterapeuta y un podólogo. Los servicios ofrecidos por estos sanitarios no tienen cobertura pública. Por último, el municipio también cuenta con una residencia de ancianos, Residencia Servicio de Teleasistencia de Lucena del Cid.

\section{Discusión y conclusiones}

Lucena del Cid es un municipio con una población envejecida, siendo la tasa de longevidad de un $53 \%$. Este hecho hace aumentar la tasa de dependencia que, en su totalidad, es del $60 \%$. Aun así, se debe tener en cuenta que existe también población juvenil, la cual también es dependiente y que supone un $17,1 \%$ del porcentaje anterior. El hecho de que haya una gran tasa de longevidad conlleva la afloración de enfermedades crónicas las cuales se deberán tratar tanto en atención primaria como en atención secundaria.

En lo referente a la situación laboral, en 2014 había un total de 130 personas en el paro de las 876 personas activas, lo que supone un 14,84\%. Al tratarse de un municipio pequeño, no se generan los suficientes puestos de trabajo para cubrir las necesidades laborales, de forma que cada vez hay más emigraciones por parte de los habitantes más jóvenes. La emigración implica que la población de Lucena del Cid disminuya, puesto que las emigraciones son mayores (52) que las inmigraciones (26) (año 2013). Este hecho, además, conlleva a que la población esté cada vez más envejecida, siendo la tasa de envejecimiento de $249,7 \%$.

En cuanto al estado de salud de la población de Lucena, se realizó un estudio para conocer cuáles son las enfermedades con mayor índice de prevalencia. Los resultados que se obtuvieron fueron los siguientes:

Las enfermedades cardiovasculares son las afecciones más prevalentes en la población lucenense $(27,166 \%)$. En lo referente a la prevención y tratamiento de estas enfermedades, el punto de atención continuada de Lucena lleva a cabo distintos programas de salud, comprendidos en el programa de salud de adultos publicado por la Conselleria de Sanitat Universal i Salut Pública (Conselleria de Sanitat, 1998), para intentar abordar de forma primaria dichas afecciones, así como la realización de controles periódicos de forma que pueda llevarse 
un control correcto de enfermedades como la hipertensión arterial. Además, aunque se trata de un municipio pequeño y no dispone de una ruta para caminar, sí que existe un camino desde Lucena hasta una ermita que se encuentra a las afueras y por donde los habitantes pueden caminar y así realizar el ejercicio diario recomendado. En Lucena del Cid, también hay varios parques, uno de ellos con maquinaria para que las personas mayores puedan ejercitarse sin necesidad de desplazarse muy lejos.

En cuanto a las necesidades de las enfermedades oncológicas, las segundas más prevalentes $(23,43 \%)$, la prevención primaria se realiza siguiendo el programa de salud de adultos. Sin embargo, es en el hospital donde se realizan las distintas técnicas de cribado para los diferentes cánceres como el de mama y el de colón, así como el tratamiento de éstas.

En tercer lugar, se encuentran las enfermedades respiratorias con una prevalencia del $7,94 \%$, cuyas necesidades quedan cubiertas con los programas de salud realizados por los profesionales sanitarios del municipio. Además, Lucena del Cid es un municipio situado a la ladera de una montaña, rodeado de vegetación y sin demasiada presencia industrial por lo que el aire es bastante limpio y sin contaminación.

Por lo que respecta a los programas de salud que se desarrollan en la población, se contactó por teléfono con el centro de salud de Lucena del Cid, y tras varios intentos se obtuvo información sobre los programas de salud que se llevan a cabo en el municipio. Éstos son el Programa de Salud del Niño Sano (Fullana, de Hevia, Jordá, Momparler, Moreno \& Redondo, 1999) y el Programa de Adultos publicado por la Conselleria de Sanitat Universal i Salut Pública (Conselleria de Sanitat, 1998). Nos comentaron que con estos programas las necesidades de la población del municipio estaban cubiertas.

Aun así, se piensan diferentes mejoras para obtener un mejor nivel de salud de la población:

1. Con respecto a las enfermedades cardiovasculares, mejorar el servicio del que se dispone creando una consulta mensual donde haya un cardiólogo que controle con mayor facilidad a los enfermos con dichas afecciones.

2. En el caso del cribado de las enfermedades neoplásicas, se hará llegar de forma anual una unidad móvil con el instrumental necesario para realizar los cribados. Esta mejora sería conveniente, debido a que las patologías oncológicas son las segundas más prevalentes en Lucena. También, se debe tener en cuenta que un 30,5\% de la población masculina del municipio tiene una edad comprendida entre los 50 y 69 años, edades en las que se realiza el cribado de colon (AECC, 2016a). En el caso de las mujeres, un $20,11 \%$ de la población se encuentra entre la franja de edad de los 50-65 años, edad a la que se realizan las mamografías. Se debe tener en cuenta también, la población femenina en la que a partir de 45 años se recomienda la realización de este cribado por presentar factores de riesgo (AECC, 2016b).

3. Por último, se cree conveniente que se mejore el transporte público y se amplíen los horarios para que la población tenga más posibilidades de acudir a los centros de especialidades y a los hospitales de Castellón de la Plana.

\section{Referencias bibliográficas}

Asociación Española contra el Cáncer. (2016a). Contra el cáncer, contigo somos más fuertes [Internet]. [consultado 2 de enero de 2017]. Disponible en:

https://www.aecc.es/msites/tusalud/Paginas/Anteriores/Marzo2016/index.html 
Asociación Española contra el Cáncer. (2016b). Diagnóstico precoz del cáncer de mama [Internet]. [consultado 5 de enero de 2017]. Disponible en:

https://www.aecc.es/SobreEICancer/CancerPorLocalizacion/CancerMama/Paginas/diagnosticoprecoz.aspx

Arenas-Monreal, L., Cortez-Lugo, M., Parada-Toro, I., Pacheco-Magaña, L., \& Magaña-Valladares, L. (2015). Population health diagnosis with an ecohealth approach. Revista De Saúde Pública, 49(0). http://dx.doi.org/10.1590/s0034-8910.2015049005842

Conselleria de Sanitat (1998). Portal Estadístico de la Generalitat Valenciana - Generalitat Valenciana. Salud del adulto [Internet]. [citado 4 de enero de 2017]. Disponible en: http:// www2.san.gva.es/cas/prof/recomendaciones.html\#1

Conselleria de Sanidad. (2016) Portal Estadístico de la Generalitat Valenciana - Generalitat Valenciana [Internet]. [consultado 30 de diciembre de 2016]. Disponible en: http://www. pegv.gva.es/

Fullana, A. M., de Hevia, R., Jordá, D., Momparler, P., Moreno, M. P., \& Redondo Gallego, M. J. (1999) Programa de supervisión de la Salud Infantil [Internet]. [consultado 4 enero de 2017], 2, 5-92

Instituto Nacional de Estadística. (2017). Instituto Nacional de Estadística. (Spanish Statistical Office). Productos y Servicios / Publicaciones / Productos y Servicios / Publicaciones /Publicaciones de descarga gratuita [Internet]. [consultado 2 de enero de 2017]. Disponible en: http://www.ine.es/ss/Satellite?L=es_ES\&c=INESeccion_C\&cid=1259926380048\&p=125 4735110672\&pagename=ProductosYServicios/PYSLayout

Lucenadelcid.es. (2016). Ajuntament de Lucena del Cid [Internet]. [acceso 23 de diciembre de 2016]. Disponible en: http://www.lucenadelcid.es/ 